(2) Open Access Full Text Article

REVIEW

\title{
How to promote and preserve eyelid health
}

This article was published in the following Dove Press journal:

Clinical Ophthalmology

24 October 2012

Number of times this article has been viewed

\section{Jose M Benitez-del-Castillo}

Ocular Surface and Inflammation, Department Ophthalmology, Hospital Clinico San Carlos, Madrid, Spain
Correspondence: JM Benitez-del-Castillo Ocular Surface and Inflammation, Department Ophthalmology, Hospital Clinico San Carlos, Madrid 28040, Spain Tel +349 I330 3963

Fax +34913303975

Email jbenitez.hcsc@salud.madrid.org
Abstract: Disorders of the lacrimal functional unit are common in ophthalmological practice, with meibomian gland dysfunction, blepharitis, and dry eye forming a significant part of the general ophthalmologist's practice. The eyelid and its associated structures form a complex organ designed to protect the fragile corneal surface and improve visual acuity. This organ is subject to a number of disorders, including meibomian gland dysfunction, dry eye syndrome, anterior blepharitis, allergic and dermatological conditions, and disorders associated with contact lens use. Although commonly described separately, disorders of the lacrimal function unit are better considered as a group of interacting pathologies that have inflammatory mediators as a central feature. Eyelid hygiene, in the sense of routine cleansing and massage of the eyelids, is well accepted in the management of many disorders of the eyelid. However, a broader concept of eyelid health may be appropriate, in which eyelid cleansing is but a part of a more complete program of care that includes screening and risk assessment, patient education, and coaching. The ophthalmologist has an important role to play in helping patients persist with routine eyelid care that may be long-term or lifelong. A number of preparations exist to make routine eyelid care both more effective and more pleasant, and might also improve compliance. Several such preparations have been devised, and are being assessed in clinical studies, and appear to be effective and preferred by patients over traditional soap and water or baby shampoo.

Keywords: eyelid, disorders, health, lacrimal functional unit

\section{Introduction}

Disorders of the eyelid are amongst the most frequently encountered pathologies in routine clinical ophthalmological practice. Meibomian gland dysfunction, blepharitis, and dry eye comprise a large portion of the workload of general practicing ophthalmologists. Treatment of these disorders is not straightforward, there are no simple answers, and much of the burden of routine eyelid care falls on the patient. The present review considers whether the currently well-understood concept of eyelid hygiene should be generalized to a broader concept of eyelid health.

\section{The healthy eyelid}

The healthy human eyelid is a remarkable but all too frequently neglected structure (Figure 1). Its anatomy and physiology are adapted to a number of specific functions, collectively crucial to the health of the eye, that include protection of the vulnerable ocular surface from physical insult and providing a lubricated and hydrated environment for movement of the eyelid and a smooth ocular surface for optimal visual acuity. ${ }^{1}$ The International Dry Eye Workshop in 2007 defined the "lacrimal functional unit" 


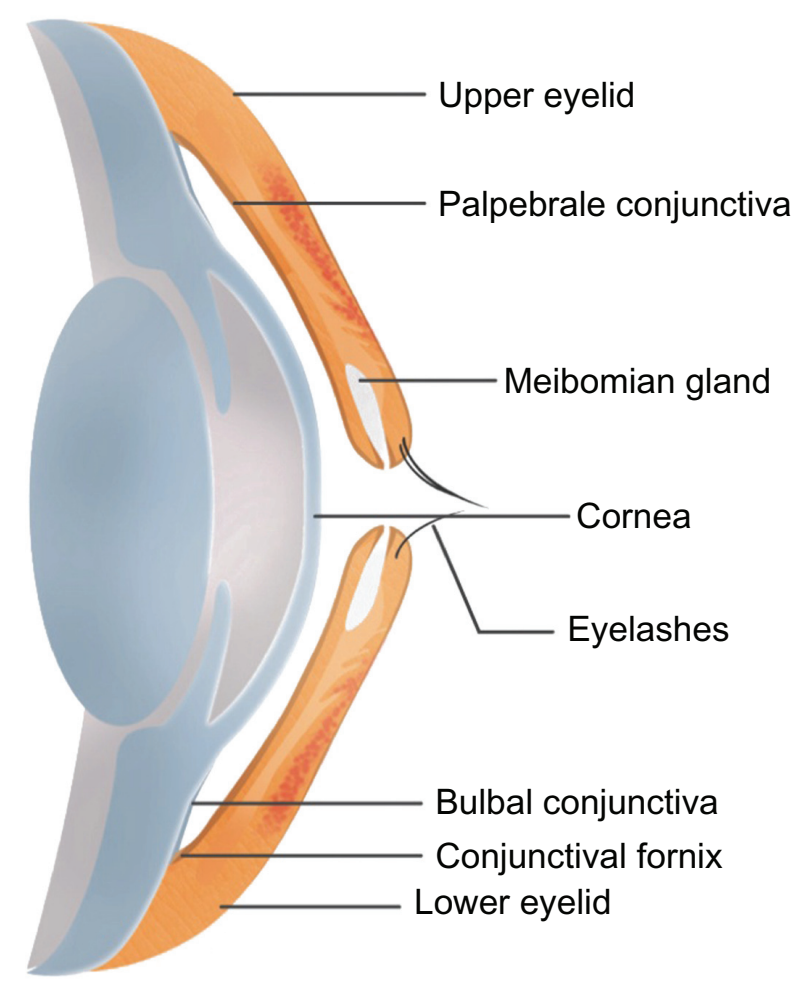

Figure I Schematic sagittal section of the lacrimal functional unit.

as an integrated structure comprising the lacrimal glands, ocular surface (cornea, conjunctiva, and meibomian glands), eyelids, and the sensory and motor nerves that connect them. ${ }^{2}$ This concept gives this functional system the prominence it deserves, given its important role in maintaining the health of the exterior optical surface, and thereby vision.

The cornea is the most fragile external structure of the body and relies entirely on the eyelid and adjacent structures to maintain its patency; a cornea directly and permanently exposed to the environment will rapidly succumb to epithelial defects, scarring, vascularization, and infection, and is experienced by the patient as irritation, pain, loss of visual acuity and, eventually, loss of sight. ${ }^{3}$ As with many ophthalmic disorders, even small degrees of dysfunction can have very significant impacts on quality of life and the ability to carry out normal daily tasks.

The healthy eyelid comprises a lamellar structure with fine skin on the outer surface and conjunctiva on the inner surface. Between these layers lie a number of muscle groups that control the movement of the eyelid, and in particular the blink reflex, as well as the tarsal plate that comprises the meibomian glands.

The tear film can be considered a substructure of the eyelid and forms a highly important layer between the eyelid and the surface of the eye and between the exterior environment and the eye surface. The tear film is predominantly aqueous in nature and is formed from the secretions of the lacrimal glands. However, although minor in quantity, the lipids in the tear film formed from the sebaceous secretions of the meibomian glands are crucial to its function. The tear film provides protective, lubricant, nutritional, and antimicrobial functions, as well as playing an important role in visual acuity. ${ }^{1,4-9}$ The physiology of the tear film is relatively complex, comprising a very thin (less than $100 \mathrm{nM}$ ) outer lipid layer that overlies an aqueous layer enriched with water-soluble proteins, electrolytes, carbohydrates, and other materials; the innermost layer is also aqueous and contains mucins. The aqueous layers are considerably thicker than the lipidic layer (around $4 \mu \mathrm{m}$ ). ${ }^{10}$ The meibomian glands (Figure 2) are responsible for production and secretion of the lipid and protein components of the tear film, the function of which is to stabilize and, most importantly, to prevent evaporation of the tear film. ${ }^{11}$ The meibomian glands are adapted sebaceous glands located on the edge of each eyelid although, unlike sebaceous glands in other parts of the body, each is not specifically associated with a hair (or eyelash) follicle. Each meibomian gland is formed from a long central duct with chains of secretory acini arranged around it in a radial pattern. The glands are arranged in a single row extending the width of the eyelid. The proteinaceous lipidic material produced, meibum, is secreted from a terminal duct onto the posterior lid margin and expressed on the ocular surface during eyelid movements. During sleep and periods of reduced blinking (eg, during visual concentration), meibum accumulates in the ducts of the gland and can be expressed in quantity by forced blinking. Production of meibum is modulated by a very large number of hormonal and neural influences, including androgens, progestin, estrogen, corticotrophin-releasing hormone, and substance $\mathrm{P}$, as well as by the autonomic nervous system. ${ }^{12}$ The relative importance of these systems in the physiology of the meibomian gland

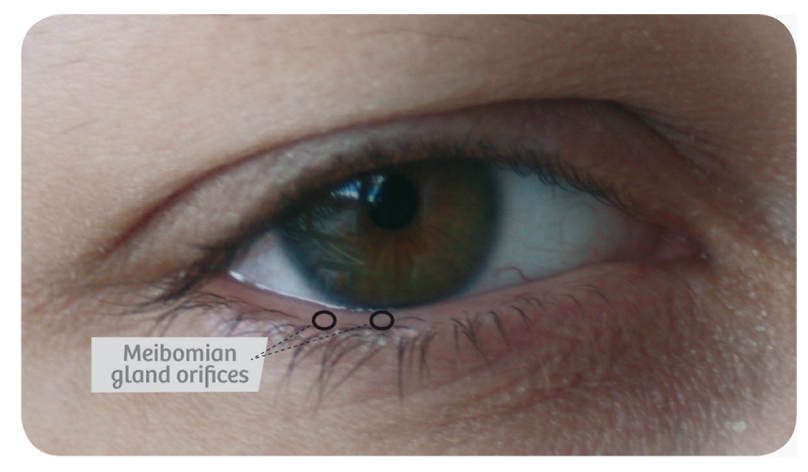

Figure $\mathbf{2}$ Location of the meibomian gland orifices. 
is not well understood, but clearly offers the opportunity for a rich control system. ${ }^{10}$

\section{Disorders of the eyelid}

The eyelid can be subject to a variety of disorders, ranging from benign and self-limiting disease to neoplasia. In the current context, we consider a related and interacting group of illnesses, the course of which could conceivably be improved by eyelid hygiene.

\section{Meibomian gland dysfunction}

According to a recent international workshop, meibomian gland dysfunction is defined as "a chronic, diffuse abnormality of the meibomian glands, commonly characterized by terminal duct obstruction and/or qualitative/quantitative changes in the glandular secretion. It may result in alteration of the tear film, symptoms of eye irritation, clinically apparent inflammation, and ocular surface disease." ${ }^{13}$ The term posterior blepharitis is often used synonymously with meibomian gland dysfunction, though in its early stages, meibomian gland dysfunction may not be associated with inflammation of the eyelid. The symptoms of meibomian gland dysfunction are the result of an impaired quantity or quality of meibum supplied to the ocular surface. Most commonly, the terminal ends of the ducts become blocked with keratinized cells. However, such obstruction of the duct can lead to ductal dilatation and loss of secretory cells in the acini of the gland. ${ }^{10,11}$ Blocked terminal ducts reduce the quantity of meibum which can be produced by the gland, and also appear to affect its lipid composition deleteriously as well, with a tendency for more branched chain fatty acids and cholesterol in the meibum, ${ }^{14}$ which gives a more waxy and viscous character (Figure 3).

Difficulties in definition and lack of standardized tools for clinical assessment have hampered systematic studies of the

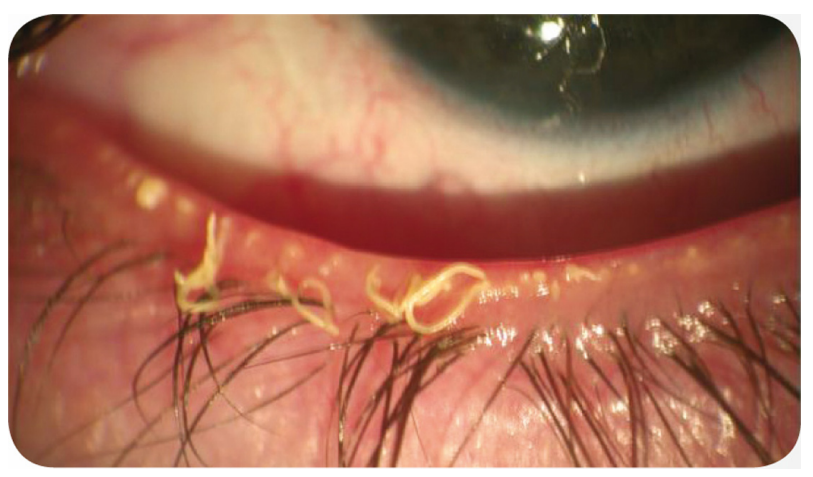

Figure 3 Meibomian gland dysfunction: strings of waxy, dysfunctional meibomian secretion. epidemiology of meibomian gland dysfunction. Moreover, the specialized tests required to identify meibomian gland dysfunction in its nonsymptomatic manifestations are not appropriate for use in population-based epidemiological studies. ${ }^{15}$ However, some trends are clear, ie, Asian populations appear to have a much higher prevalence of meibomian gland dysfunction (up to 60\% in some studies) than do Caucasians (typically 3\%-20\%). ${ }^{15}$ A number of factors have been identified which coexist with meibomian gland dysfunction and, whilst causal links have not been proven, plausible mechanisms exist for connecting them with the pathophysiology of meibomian gland dysfunction, ie, anterior blepharitis, contact lens use, Demodex mite infestation, and dry eye disease. ${ }^{15}$ In addition, hormonal conditions such as menopause and androgen deficiency might contribute to the illness, as could rosacea, psoriasis, atopy, and hypertension. ${ }^{15}$

Clinical manifestations of meibomian gland dysfunction can range from the barely perceptible to serious and sight-threatening changes in the ocular epithelium. ${ }^{16}$ The predominant symptoms are related to dry eye, of which meibomian gland dysfunction is a major cause ${ }^{17}$ Eyelid hygiene is considered the mainstay of clinical treatment for meibomian gland dysfunction. ${ }^{18}$ Reliable and controlled heating will melt meibum and facilitate its release by massage and cleansing.

\section{Dry eye}

Dry eye is one of the most frequently encountered ocular complaints in general ophthalmological practice. ${ }^{19}$ Formally defined by the International Dry Eye Workshop as "a multifactorial disease of the tears and ocular surface that results in symptoms of discomfort, visual disturbance, and tear film instability with potential damage to the ocular surface. It is accompanied by increased osmolarity of the tear film and inflammation of the ocular surface."2 However, its true prevalence remains obscure. Whilst there is no shortage of epidemiological studies offering estimates of prevalence, ranging from less than $0.1 \%{ }^{20}$ to more than $15 \%,{ }^{21}$ methodological differences and a lack of uniform diagnostic criteria ${ }^{12}$ have hampered attempts at establishing the true prevalence. ${ }^{22}$ Nevertheless, most practicing ophthalmologists would tend towards an estimate at the higher end of this range.

Dry eye syndromes are classified into two major categories, ie, aqueous-deficient, in which a diminution in the amount of tears produced is the primary etiology, and evaporative, in which aqueous tear production is adequate, but evaporation reduces the effectiveness of the tear film. ${ }^{2}$ Aqueous-deficient dry eye disease may be subdivided into 
Sjögren or non-Sjögren, the former being an autoimmune disease of the lacrimal and salivary glands and the latter being due to various disorders of the lacrimal functional unit, such as lacrimal gland insufficiency or ductal obstruction. ${ }^{2}$ Evaporative dry eye disease can be a consequence of meibomian gland dysfunction as well as allergic conjunctivitis, and anatomical disorders of the lacrimal functional unit or the blink reflex. ${ }^{12}$ In recent years, there has been considerable interest in the role of inflammatory mediators in the initiation and maintenance of dry eye and a plausible hypothesis exists in which changes in tear composition initiate the release of inflammatory mediators that in turn alter tear composition. ${ }^{23}$

The clinical presentation is variable and can include eye irritation of varying degrees of severity and persistence, pain, ocular fatigue, and blurred vision. ${ }^{24}$ The clinical course is typically chronic, and whilst patients can obtain some degree of relief with appropriate treatment, a definitive cure is rarely obtained. ${ }^{12,25}$ The addition of an eyelid hygiene regimen decreased corneal epithelial permeability more than tear lubricants alone in patients with Sjögren syndrome in a 2-week study. ${ }^{26}$

\section{Allergy, dermatological conditions, and contact lenses}

The eye provides an excellent medium for the expression of allergic symptomatology, as well as pathology caused by contact lens use and other environmental factors. Many skin disorders also have an ocular component. Manifestations of this group of disorders can range from the commonplace, such as allergic conjunctivitis, to the rare and spectacular, such as giant papillary conjunctivitis. ${ }^{15}$ Allergic conjunctivitis is a common disorder, with recent studies finding prevalence rates as high as $40 \%$, and the cardinal signs and symptoms being itching, redness, and eyelid swelling. ${ }^{27}$ Though generally self-limiting, both seasonal and perennial forms of allergic conjunctivitis cause considerable discomfort and have profound effects on quality of life. ${ }^{27}$ Both seasonal and perennial forms of allergic conjunctivitis share a pathology involving a classical immunoglobulin E/ mast cell-mediated reaction to airborne allergens (typically pollen in the seasonal form, and mites, mold, and animal dander in the perennial form). ${ }^{28}$ Ocular symptoms usually exist in common with nasal symptoms in allergic rhinitis and contribute significantly to the burden of illness, to the extent that the term rhinoconjunctivitis has been coined to represent better the clinical manifestations and course of the disorder. ${ }^{27,28}$
Contact lens use is associated with meibomian gland dysfunction, and dry eye. Eyelid hygiene should result in increased contact lens tolerance by improving meibomian gland function (and thus decreasing evaporation) and dry eye, and improving giant papillary conjunctivitis. Eyelid hygiene will help remove allergens from the lid margin, decreasing its access to the conjunctiva.

\section{Anterior blepharitis}

Anterior blepharitis is a chronic inflammation of the eyelid margin. It is extremely common worldwide, and indeed is probably the most common presentation in routine ophthalmologic practice. ${ }^{29,30}$ As well as inflammation, typical symptoms include irritation, burning sensation, foreign object sensation, tearing, and dry eyes. Frequently patients will complain of their eyelids being stuck together upon wakening. Anterior blepharitis involves the anterior lid margin and eyelashes, and is associated with staphylococcal infection ${ }^{31}$ or Demodex mite infestation. ${ }^{32}$ Certain dermatological conditions, such as seborrheic dermatitis, rosacea, and eczema carry an increased risk of anterior blepharitis. ${ }^{12}$ In any case, the clinical course can vary from mild and self-limiting to chronic, with lid margin hypertrophy, scarring, madarosis, trichiasis, and poliosis. ${ }^{33}$ Treatment is frequently unsatisfactory, and requires considerable commitment from the patient to regular, long-term, and rigorous eyelid hygiene regime. ${ }^{33-35}$

\section{Interacting pathologies}

It is clear from the brief foregoing description of the various eyelid pathologies that there is a considerable degree of interaction between these conditions. Meibomian gland dysfunction and inflammation are pathophysiological processes that can be identified as cause or consequence in many of the illnesses described above. Meibomian gland dysfunction, for example, can result in tears that evaporate too quickly and result in dry eye and blepharitis. The increasing evidence of an inflammatory component in dry eye also points in a similar direction. ${ }^{2,36}$

Altered meibomian lipids ${ }^{37}$ produced in meibomian gland dysfunction may themselves be proinflammatory or be transformed into proinflammatory compounds by bacteria. In any event, the altered lipids are less easily expressed from the glands due to their waxy nature (more ordered conformation) and are less effective at providing the hydrophobic barrier the tear film requires to avoid excessive evaporation. A scheme for understanding disorders of the ocular surface and conjunctiva as a related and interacting group of diseases and risk factors has been proposed..$^{38,39}$ 


\section{Eyelid hygiene or eyelid health?}

Arriving in the English language via the latinization of a Greek root, hygiene means conditions and practices that serve to promote or preserve health. ${ }^{40}$ Hygeia was the daughter of the Greek god of medicine Askiepios, and like her sister, Panaceia, she followed her father into medicine, but was specifically charged with the prevention of illness and the promotion of health. ${ }^{41}$

Although the distinction between hygiene and health can be obscure, the concept of hygiene commonly refers to the prevention of disease via practices in normal daily life, rather than the treatment of illness or risk factors for disease. The concept of dental hygiene is well understood, but, for example, the idea of "lipid hygiene" maintained with statins is less readily appreciated despite the widespread use of these drugs.

In modern usage, hygiene implies promotion of health through prevention of infection, particularly through cleaning regimes. Although cleansing is an important component of eyelid health, there is a case to define a wider concept of eyelid health to include screening and patient education as well as warming, massage, and cleansing routines.

An analogy between eyelid health and dental health is apposite. The dental patient is responsible for cleaning their teeth, but regular screening (and, if necessary, intervention) by both dentist and dental hygienist identifies the development of gum problems at as early a stage as possible. It has become routine practice for the dental hygienist to educate their patients on the importance of healthy gums as well as in techniques for maintaining gum health. They also routinely recommend products (devices, such as electric toothbrushes, interdental sticks, as well as consumables, such as toothpaste) both to improve gum health and to make the hygiene process pleasant and effective, which in turn improves compliance with the hygiene regime.

Similarly, an analogous concept of eyelid health that incorporates screening and risk assessment, patient education, daily hygiene regimes, and treatment intervention when necessary seems appropriate in this context. Moreover, whilst the symptoms of these disorders are unpleasant in themselves and in some cases debilitating, they can also lead to more serious, sight-threatening conditions if left untreated; blepharitis for example, can lead to conjunctivitis and permanent lid margin changes, such as meibomian gland dropout, marginal keratitis, corneal neovascularization, and cicatricial lid changes. ${ }^{42}$ Blepharitis is also a risk factor for endophthalmitis after cataract surgery. ${ }^{43,44}$ Blepharitis has been reported in as many as $60 \%$ of patients about to undergo cataract surgery. ${ }^{45}$ Reducing blepharitis, and consequently tear film insufficiency, would reduce the bacterial colonization of the ocular surface that can result in postoperative ocular infections. ${ }^{46}$

\section{Components of eyelid health Warming, massage, and cleansing}

The ocular surface (as part of the lacrimal functional unit) is an anatomically complex structure and its physical location, surrounded by nose, cheeks, and brows, means that it is not as readily accessible to routine daily cleaning as the surrounding structures. ${ }^{32}$ The aqueous environment of the ocular surface and eyelids and the proteinaceous/lipidic secretions of the meibomian glands form a convenient locus for infection by Staphylococci and infestation by Demodex species. The accumulation of crusts on the eyelashes and eyelid margin also encourages infection and infestation.

The principle that pathologically altered meibomian lipids are melted by warming is sound, but when used in clinical practice, there is a risk that its efficacy may be compromised by poorly standardized procedures (variable duration and degree of warming as well as imperfect compliance) ${ }^{47}$ Specific devices have been developed to assist the patient in delivering moist heat therapy in a consistent and effective manner. ${ }^{48}$ Once Meibomian secretions are melted, massage helps in relieving meibomian gland obstruction.

Cleansing is a well-established part of the treatment of anterior blepharitis, but likely has benefits for other pathologies as well. The effective removal of crusts around the eyelid margin reduces the possibility of bacterial infection that not only contributes directly to anterior blepharitis, but also has a deleterious effect on meibomian gland function..$^{49}$ In addition, cleansing, done in combination with massage of the eyelid, has multifactorial benefits on the function of the lacrimal functional unit, that includes encouraging expression of meibum, especially from blocked or partially blocked ducts which have thicker, more waxy meibum, an effect that is enhanced if the eyelid is warmed during the process to reduce the waxiness and increase the fluidity of meibomian gland secretions. This process will in turn improve the stability of the lipid layer, with consequent benefits for the patient with dry eye and meibomian gland dysfunction.

\section{Screening and risk assessment}

Screening of patients could also be an important method for identifying early cases of lacrimal functional unit disorders. Epidemiological studies suggest that a considerable number of patients attending ophthalmology practices have clinical 
signs of blepharitis, even where this is not the reason for the consultation. ${ }^{30}$ Likewise, the prevalence of dry eye disease was found to be $14.4 \%$ overall, rising to $19 \%$ in those over 80 years of age ${ }^{21}$ and to be diagnosed in twice as many patients by Schirmer's test as by reported symptoms. ${ }^{50}$ These findings suggest that there are undiagnosed cases of these illnesses that may be revealed by screening. Early intervention can prevent worsening of symptoms and the longer-term sequelae of the illness. ${ }^{51}$ Therefore, a wider concept of eyelid health should include screening for disorders of the lacrimal functional unit by clinical examination and questioning for symptoms and signs, particularly in patients who may be at higher risk (Table 1).

Although the causal links are not always clear, there is now sufficient empirical evidence to identify patients who are likely to be at risk from disorders of the lacrimal functional unit and who are therefore most likely to benefit from measures aimed at improving their eyelid health.

The increased susceptibility of contact lens users to a variety of disorders, from mild blepharitis to infectious keratitis, is well known. ${ }^{52,53}$ Increased use of the Internet for repeat purchase of contact lenses has resulted in less frequent contact with ophthalmologists and an increased risk of associated pathology ${ }^{54}$ Given that rigorous eyelid hygiene has been shown to reduce the risk of cancellation of cataract surgery due to blepharitis, this group of patients may also be a useful target for intervention with eyelid health practices. ${ }^{55}$

\section{Patient education}

Patient education on the importance of maintaining a healthy tear film and the role of the eyelids in overall eye health can help compliance with a long-term, perhaps lifelong, commitment to eyelid health. Patients can be instructed on how to clean and massage the eyelid to promote its function, how to avoid exacerbating factors, but most of all, encouragement to continue cleaning and massage routines and active follow-up will help them to maintain these routines. Patients can also be helped with the recommendation of products that make such hygiene procedures both effective and pleasant.

Table I Screening factors for development of eyelid disorders

Existing ocular disorder
Contact lens use
Rosacea and other skin disorders
Age
Existing systemic illness (especially diabetes, arthritis, thyroid disease) and
some specific pharmacological treatments
Sex hormone treatment or disorder (androgen deficiency, androgen
therapy, and menopause, for example)

Soap and water washing does not have the effect on bacterial colonization of the eyelids that might be expected, ${ }^{56}$ and in any case, effective cleansing of the eyelid margin is difficult to achieve with soap and water because of discomfort of soap in the eyes and likely harm to the tear lipid layer. Maintaining compliance during a long-term, effective eyelid hygiene regime with soap or baby shampoo is at best difficult (due to stinging) and at worst harmful (damage to the eyelid lipid layer).

Preparations for long-term eyelid hygiene need to fulfill a number of criteria, as outlined in Table 2. Although a number of eyelid scrubs and eyelid cleansing systems exist and patients appear to prefer them to simple soap or diluted shampoos, ${ }^{57}$ rather few have been submitted to any form of clinical examination in eyelid disorders, though some have been tested in the context of surgical interventions, rather than routine day-to-day eyelid care. ${ }^{55,58}$ Such eyelid cleansing products exist in a variety of presentations, ie, solutions for dissolving crusts, gels that encourage mechanical removal, and ready-to-use pads and foams. Patients can be assisted to select products that are most appropriate for their situation.

\section{Eyelid cleansing procedures}

The objective of eyelid cleansing is not only to remove crust and debris from the lid margin, but also to express meibum from the meibomian glands that may be partially or completely blocked or be obstructed by thicker than normal or waxy secretions. The procedure will differ in detail depending on the medium and product used, but the general principles are as follows (Figure 4):

- A hot (not too hot) dampened compress or face cloth (the compress or applicator supplied with a specific eyelid cleansing system), should be held against the closed eye for around 5 minutes. This loosens scales on the eyelid margin and improves the fluidity of meibum. However, warm compresses are a poorly standardized treatment and nowadays new eyelid warming devices provide constant and controlled moist heat therapy. ${ }^{59}$

- There are a number of techniques for lid massage, which helps express blocked meibomian glands. In general, the massage should proceed from the root of the eyelid to

Table 2 Properties of an ideal eyelid cleanser

\begin{tabular}{ll}
\hline Property & Benefit \\
\hline Effective removal of eyelid crusts & Clinical improvement \\
Pleasant and easy to use & Encourage long-term compliance \\
No preservative, parabens, perfume & Avoid secondary effects \\
or low toxicity alternatives where & \\
preservatives are unavoidable & \\
\hline
\end{tabular}



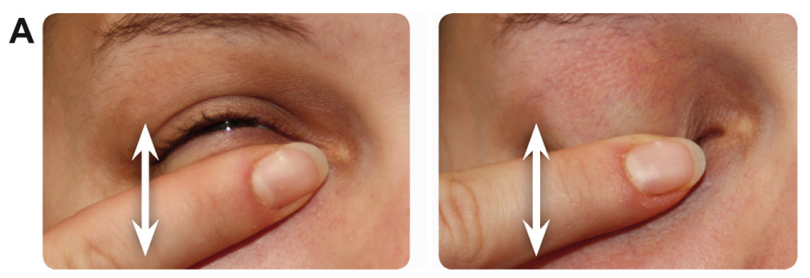

B
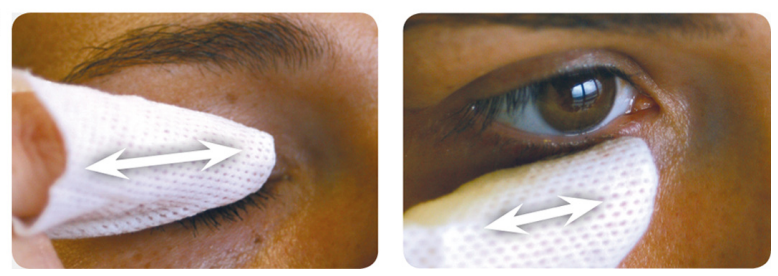

Figure 4 (A) Eyelid cleaning procedure: eyelid massage to express waxy meibomian secretion. (B) Eyelid cleaning procedure: cleaning of eyelid margin.

the margin using a warm compress or a gentle pinching action on the eyelid, again from root to margin.

- The lid margin should be cleaned by gentle rubbing with a cotton bud or other applicator on the lid margin and just inside the lid, avoiding contact with the eye.

Traditionally compresses, face towels, and baby shampoo have been the mainstay of eyelid cleansing, but nowadays a number of eyelid cleaning systems have been developed to aid the process and make it more comfortable and acceptable. Improved acceptability encourages the continued long-term compliance required.

\section{Clinical studies with eyelid cleansers}

Although the use of compresses and face towels in eyelid hygiene procedures is widespread, it is based more on extended clinical experience than specific clinical trials. ${ }^{60}$ However, there are clinical trials with the newer eyelid hygiene systems, albeit not controlled trials. An eyelid scrub known as I-Scrub (currently discontinued), the precise formulation of which is now obscure following the demise of the company (Spectra Pharmaceuticals Hanover, MA) ${ }^{61}$ was the subject of a single small study in 20 patients with chronic blepharitis, the results suggesting that it was well accepted, improved their condition, and did not result in any deterioration in the patient's condition. ${ }^{62}$

A significantly larger, prospective, randomized, doubleblind study in 286 patients sought to assess the efficacy of Blephasol $^{\circledR}$ (Laboratoires Théa, Clermont Ferrand, France) acapriloglycine-containing eyelid cleanser in reducing conjunctival flora before cataract surgery. Although the number of positive cultures was not reduced to a statistically significant extent, treatment for 4-5 days reduced the number of different organisms present (Staphylococci being almost the only species). ${ }^{61}$
Blephaclean $^{\circledR}$ (Laboratoires Théa) is an eyelid-cleansing system comprising wipes impregnated with a lipid micellecontaining aqueous medium, which can be used to clean the eyelids without rinsing or wiping afterwards. In a prospective, open-label study of 40 subjects with dry eye and mild-to-moderate anterior blepharitis or meibomian gland dysfunction, there were significant reductions in eyelash contamination and meibomian gland dysfunction, as well as an improvement in the quality of meibomian secretion. ${ }^{63}$

Blephagel ${ }^{\circledR}$ (Laboratoires Théa) is a cosmetic, poloxamercontaining gel designed specifically for eyelid cleansing in people with sensitive skin or eyes and in contact lens users. The great majority of such subjects found the treatment acceptable and efficient for day-to-day eyelid hygiene procedures (Figure 5). In addition, assessment by an oph-
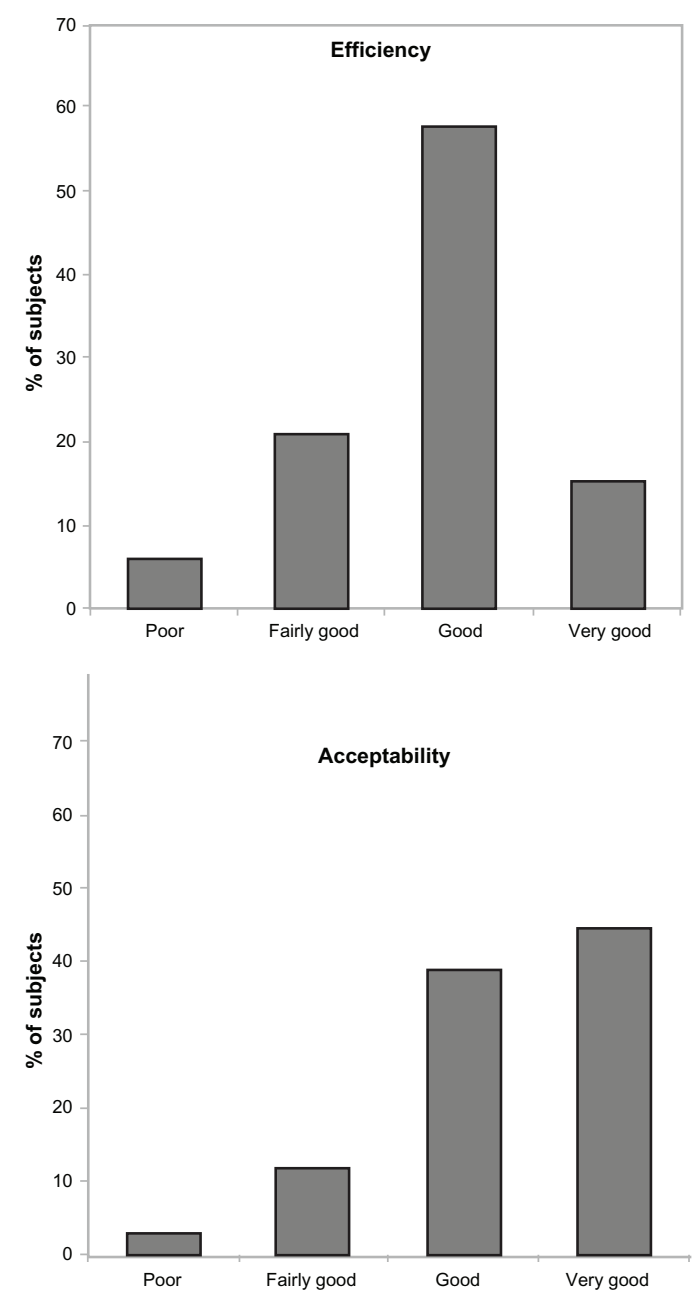

Figure 5 Appraisal of efficiency and acceptability by subjects in the presence of the investigating ophthalmologist.

Notes: Acceptability and efficiency (as a "cleaning product for eyelids and eyelashes") of the product was assessed by a self-administered questionnaire (on a 5-point ordinal scale: nil, poor, fairly good, good, very good). Reprinted from Clin Ophthalmol. (6). Doan S. Tolerability and acceptability of Blephagel: a novel eyelid hygiene aqueous gel. 6:7I-77. (C) Copyright (20I2), with permission from Dove Medical Press Ltd. ${ }^{64}$ 


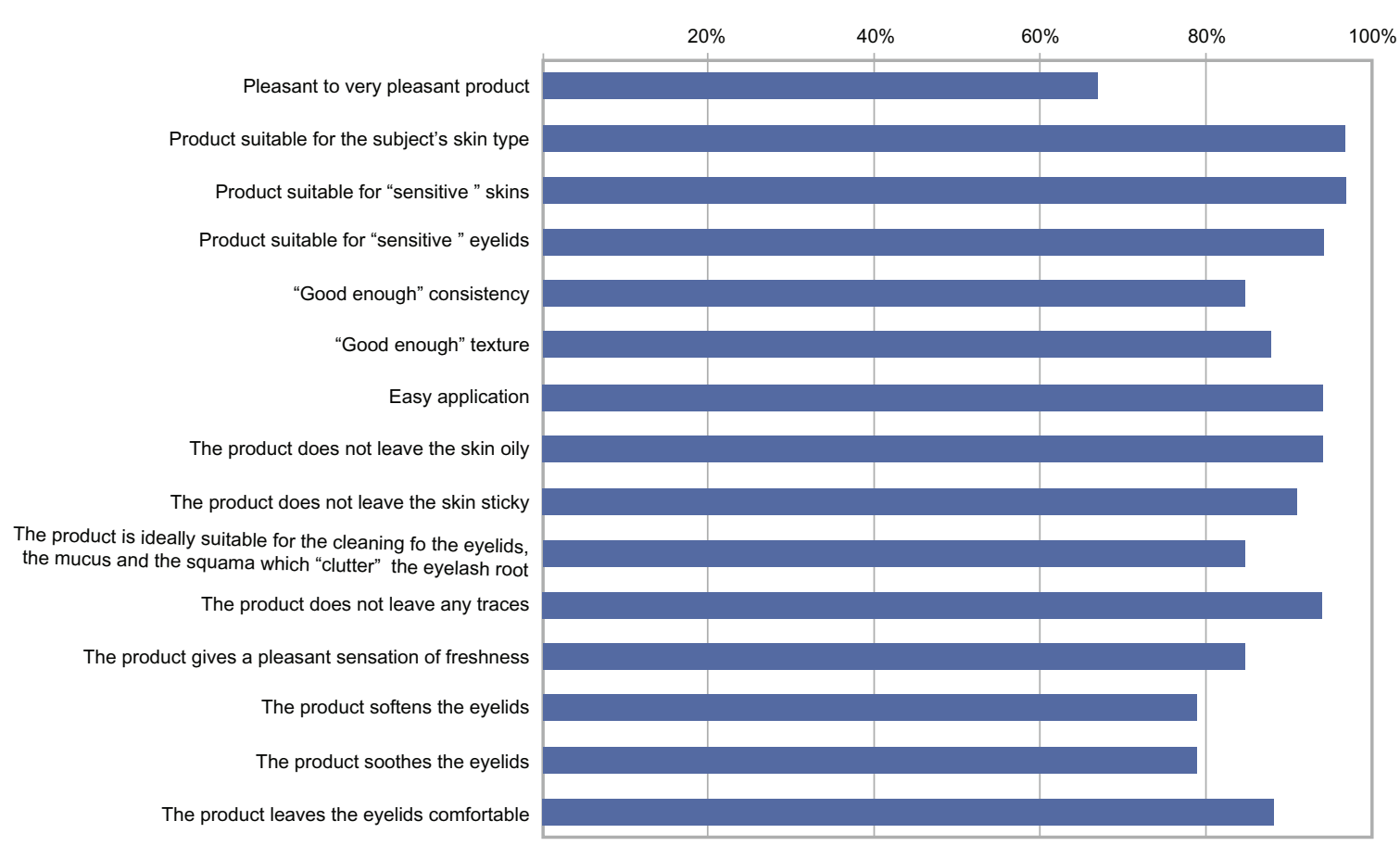

Figure 6 Subject appraisal of the qualities and efficacy of Blephagel ${ }^{\circledR}$. Reprinted from Clin Ophthalmol. (6). Doan S. Tolerability and acceptability of Blephagel: a novel eyelid hygiene aqueous gel. 6:7I-77. (C) Copyright (2012), with permission from Dove Medical Press Ltd. ${ }^{64}$

thalmologist revealed no issues with lacrimal film break-up time or visual acuity following use of the product, and the great majority of subjects endorsed the properties of the gel (Figure 6) ${ }^{64}$ Blephagel may be a useful and convenient preparation for use in daily eyelid hygiene regimes for patients with blepharitis.

A linalool-hinokitiol-based eyelid cleanser was very effective in reducing microorganisms known to cause endophthalmitis. The eyelid cleanser was more effective than povidone-iodine against all organisms. ${ }^{65}$

A comparative study of soap versus an eyelid scrub revealed that the large majority of patients preferred an eyelid scrub to soap and water. Twenty-six patients with signs and symptoms of blepharitis, the majority of whom were contact lens users, compared soap and water in one eye and an eyelid scrub in the other over a 4-month trial period. Although both cleansing regimens improved symptoms and slit-lamp findings, 17 of 25 patients completing the trial period preferred the eyelid scrub whilst only two of 25 preferred soap. ${ }^{60}$

Although the clinical evidence for eyelid cleansing products is not of the standard usually expected for pharmaceutical products, at least some of these products have been studied clinically, are effective at cleaning the eyelid, and are preferred by patients above baby shampoo and soap. In this context, they form a useful tool for the ophthalmologist who is encouraging their patients to persist with eyelid hygiene regimens.

\section{Conclusion}

The lacrimal function unit is subject to a number of interacting functional disorders, many of which have a chronic course and necessitate long-term intervention and follow-up. Clearly, the ophthalmologist plays an important role in identifying and screening patients at risk and instituting treatment appropriate to the stage or type of disease. Given the high prevalence of eyelid disorders, examination of the eyelid should be a first step in routine ophthalmological examination. Treatment is required not only to reduce symptoms and improve patient comfort, but also to prevent, delay, or avoid early reliance on antibiotics and the more serious sequelae of the disorders. Blepharitis, meibomian gland dysfunction, and dry eye are not disorders that are easily and quickly resolved by simple prescription. Long-term, perhaps lifelong, daily eyelid hygiene is required by the patient, and the ophthalmologist needs to adopt a coaching role as well as a medical role to maintain compliance in the long term. Patients need education to understand their disorder and encouragement to persist with daily eyelid hygiene. If patients understand the underlying mechanism of eyelid cleaning and massage, they are more likely to comply with it. Eyelid hygiene can also be considered as a preventative means to maintain eyelid health. Patients also 
need help in selecting the most appropriate eye care products to assist their daily regime, and the ophthalmologist can suggest preservative-free products which suit the particular lifestyle of the patient and which make the daily routine of eyelid hygiene easier and more effective.

\section{Acknowledgment}

JF Stolz provided assistance with preparation of the manuscript.

\section{Disclosure}

The authors report no conflicts of interest in this work.

\section{References}

1. Rieger G. The importance of the precorneal tear film for the quality of optical imaging. Br J Ophthalmol. 1992;76(3):157-158.

2. [No authors listed]. Research in dry eye: report of the Research Subcommittee of the International Dry Eye Workshop (2007). Ocul Surf. 2007;2:179-193.

3. Carter SR. Eyelid disorders: diagnosis and management. Am Fam Physician. 1998;57(11):2695-2702.

4. Bron AJ, Tiffany JM, Gouveia SM, Yokoi N, Voon LW. Functional aspects of the tear film lipid layer. Exp Eye Res. 2004;57(11):347-360.

5. Holly FJ. Tear film physiology. Am J Optom Physiol Opt. 1980;57(4): 252-257.

6. Mishima S, Maurice DM. The oily layer of the tear film and evaporation from the corneal surface. Exp Eye Res. 1961;1:39-45.

7. Tiffany JM. The role of meibomian secretion in the tears. Trans Ophthalmol Soc U K. 1985;104(4):396-401.

8. Tiffany JM. The lipid secretion of the meibomian glands. Adv Lipid Res. 1987;22:1-62.

9. Liu Z, Pflugfelder SC. Corneal surface regularity and the effect of artificial tears in aqueous tear deficiency. Ophthalmology. 1999;106(5): 939-943.

10. Knop E, Knop N, Millar T, Obata H, Sullivan DA. The international workshop on meibomian gland dysfunction: report of the subcommittee on anatomy, physiology, and pathophysiology of the meibomian gland. Invest Ophthalmol Vis Sci. 2011;52(4):1938-1978.

11. Mathers WD, Lane JA. Meibomian gland lipids, evaporation, and tear film stability. Adv Exp Med Biol. 1998;438:349-360.

12. Perry HD. Dry eye disease: pathophysiology, classification, and diagnosis. Am J Manag Care. 2008;14(Suppl 3):S79-S87.

13. Nelson JD, Shimazaki J, Benitez-del-Castillo JM, et al. The international workshop on meibomian gland dysfunction: report of the definition and classification subcommittee. Invest Ophthalmol Vis Sci. 2011;52(4):1930-1937.

14. Joffre C, Souchier M, Gregoire S, et al. Differences in meibomian fatty acid composition in patients with meibomian gland dysfunction and aqueous-deficient dry eye. Br J Ophthalmol. 2008;92(1): $116-119$

15. Schaumberg DA, Nichols JJ, Papas EB, Tong L, Uchino M, Nichols KK The international workshop on meibomian gland dysfunction: report of the subcommittee on the epidemiology of, and associated risk factors for, MGD. Invest Ophthalmol Vis Sci. 2011;52(4): 1994-2005.

16. Shimazaki J, Sakata M, Tsubota K. Ocular surface changes and discomfort in patients with meibomian gland dysfunction. Arch Ophthalmol. 1995;113(10):1266-1270.

17. Mathers WD, Daley TE. Tear flow and evaporation in patients with and without dry eye. Ophthalmology. 1996;103(4):664-669.

18. Nichols KK, Foulkes GN, Bron AH, et al. The international workshop on meibomian gland dysfunction: executive summary. Invest Ophthalmol Vis Sci. 2011;52(4):1922-1929.
19. O'Brien PD, Collum LM. Dry eye: diagnosis and current treatment strategies. Curr Allergy Asthma Rep. 2004;4(4):314-319.

20. Clegg JP, Guest JF, Lehman A, Smith AF. The annual cost of dry eye syndrome in France, Germany, Italy, Spain, Sweden and the United Kingdom among patients managed by ophthalmologists. Ophthalmic Epidemiol. 2006;13(4):263-274.

21. Moss SE, Klein R, Klein BE. Prevalence of and risk factors for dry eye syndrome. Arch Ophthalmol. 2000;118(9):1264-1268.

22. Brewitt H, Sistani F. Dry eye disease: the scale of the problem. Surv Ophthalmol. 2001;45 Suppl 2:S199-S202.

23. Paiva CS, Pflugfelder SC. Rationale for anti-inflammatory therapy in dry eye syndrome. Arq Bras Oftalmol. 2008;71(Suppl 6):89-95. Portuguese.

24. Lemp MA. Report of the National Eye Institute/Industry workshop on clinical trials in dry eyes. CLAO J. 1995;21(4):221-232.

25. Pflugfelder SC. Prevalence, burden, and pharmacoeconomics of dry eye disease. Am J Manag Care. 2008;14(Suppl 3):S102-S106.

26. Benitez-del-Castillo JM, Aranguez C, Garcia-Sanchez J. Corneal epithelial permeability and dry eye treatment. Adv Exp Med Biol. 2002;506 (Pt A): 703-706.

27. Rosario N, Bielory L. Epidemiology of allergic conjunctivitis. Curr Opin Allergy Clin Immunol. 2011;11(5):471-476.

28. Leonardi A, Motterle L, Bortolotti M. Allergy and the eye. Clin Exp Immunol. 2008;153 Suppl 1:17-21.

29. Lemp MA, Nichols KK. Blepharitis in the United States 2009: a surveybased perspective on prevalence and treatment. Ocul Surf. 2009;7(Supp12): S1-S14.

30. Benitez del Castillo Sanchez JM, Del Rio Novo MT, Garcia-Sanchez J. Frecuencia de la blefaritis en la consulta oftalmologica diaria. (Blepharitis prevalence in dairy ophthamic practice). St Ophthal. 1999;XVIII(3):225-230. Spanish.

31. Dougherty JM, McCulley JP. Comparative bacteriology of chronic blepharitis. Br J Ophthalmol. 1984;68(8):524-528.

32. Liu J, Sheha H, Tseng SC. Pathogenic role of Demodex mites in blepharitis. Curr Opin Allergy Clin Immunol. 2010;10(5):505-510.

33. Bernardes TF, Bonfioli AA. Blepharitis. Semin Ophthalmol. 2010;25(3): 79-83.

34. Jackson WB. Blepharitis: current strategies for diagnosis and management. Can J Ophthalmol. 2008;43(2):170-179.

35. Smith RE, Flowers CW. Chronic blepharitis: a review. CLAO J. 1995;21(3):200-207.

36. Baudouin C. Dry eye: an inflammatory disease? J Fr Ophtalmol. 1999;22(8):831. French.

37. Borchman D, Foulks GN, Yappert MC, et al. Human meibum lipid conformation and thermodynamic changes with meibomian gland dysfunction. Invest Ophthalmol Vis Sci. 2011;52(6):3805-3817.

38. Baudouin C. A new approach for better comprehension of diseases of the ocular surface. J Fr Ophtalmol. 2007;30(3):239-246. French.

39. Bowman RW, Dougherty JM, McCulley JP. Chronic blepharitis and dry eyes. Int Ophthalmol Clin. 1987;27(1):27-35.

40. Merriam-Webster Dictionary online. Available from: http://www. merriam-webster.com/dictionary/hygiene. Accessed August 17, 2012.

41. Webster's Online Dictionary. Available from: http://www.webstersonline-dictionary.org/definitions/Panacea. Accessed August 17, 2012.

42. Farrant S. Latest developments in lid management. Optician. November 10, 2011.

43. Sparrow JM. Monte-Carlo simulation of random clustering of endophthalmitis following cataract surgery. Eye. 2007;21(2):209-213.

44. Speaker MG, Milch FA, Shah MK, Eisner W, Kreiswirth BN. Role of external bacterial flora in the pathogenesis of acute postoperative endophthalmitis. Ophthalmology. 1991;98(5):639-649.

45. Luchs J, Buznego C, Trattler W. Incidence of blepharitis in patients undergoing phacoemulsification. Poster presented at the American Society of Cataract and Refractive Surgery Symposium and Congress, April 9-14, 2010, Boston, MA.

46. Goldberg DF. Preoperative evaluation of patients before cataract and refractive surgery. Int Ophthalmol Clin. 2011;51(2):97-107. 
47. Geerling G, Tauber J, Baudouin C, et al. The international workshop on meibomian gland dysfunction: report of the subcommittee on management and treatment of meibomian gland dysfunction. Invest Ophthalmol Vis Sci. 2011;52(4):2050-2064.

48. Pult H, Riede-Pult BH, Purslow C. Warming efficiency of warm compresses versus Blephasteam ${ }^{\circledR}$ in eyelid therapy. Eur J Ophthalmol. April 14, 2011. [Epub ahead of print.]

49. [No authors listed]. Management and therapy of dry eye disease: report of the Management and Therapy Subcommittee of the International Dry Eye Work Shop (2007). Ocul Surf. 2007;5(2):163-178.

50. McCarty CA, Bansal AK, Livingston PM, Stanislavsky YL, Taylor HR. The epidemiology of dry eye in Melbourne, Australia. Ophthalmology. 1998;105(6):1114-1119.

51. American Academy of Ophthalmology. Preferred Practice Patterns ${ }^{\circledR}$ Guidelines: Blepharitis. San Francisco, CA: American Academy of Ophthalmology; 2008.

52. Schlingemann RO, Nieuwendaal CP. Eye problems due to contact lenses; an advisory report from the Health Council of The Netherlands. Ned Tijdschr Geneeskd. 2002;146(35):1616-1619. Dutch.

53. Cronin B, Todd B, Lee G. The red eye in contact lens wearers - a high risk presentation. Aust Fam Physician. 2007;36(10):831-832.

54. Fogel J, Zidile C. Contact lenses purchased over the Internet place individuals potentially at risk for harmful eye care practices. Optometry. 2008;79(1):23-35.

55. Stead RE, Stuart A, Keller J, Subramaniam S. Reducing the rate of cataract surgery cancellation due to blepharitis. Eye (Lond). 2010;24(4):742.

56. Bekibele CO, Kehinde AO, Ajayi BG. Effect of face washing with soap and water and cleaning with antiseptics on upper-lid bacteria of surgical eye patients. Can J Ophthalmol. 2010;45(6):637-641.
57. Key JE. A comparative study of eyelid cleaning regimens in chronic blepharitis. CLAO J. 1996;22(3):209-212.

58. Hueso Abancens JR, Verdu E, Shargel Palacios K, et al. Modification of the conjunctival flora with cleaning palpebral solutions. Arch Soc Esp Oftalmol. 2004;79(12):617-622.

59. Villani E, Magnani F, Canton V, et al. Heating wet chamber goggles $\left(\right.$ Blephasteam ${ }^{\circledR}$ ) in meibomian gland dysfunction unresponsive to warm compress treatment. Poster abstract $602 / A 70$ presented at the Association for Research in Vision and Ophthalmology Annual Congress, May 6-10, 2012, Fort Lauderdale, FL.

60. Leibowitz HM. Corneal Disorders: Clinical Diagnosis and Management. Philadelphia, PA: WB Saunders; 1982.

61. Polack FM, Goodman DF. Experience with a new detergent lid scrub in the management of chronic blepharitis. Arch Ophthalmol. 1988;106(6):719-720.

62. Avisar R, Savir H, Deutsch D, Teller J. Effect of I-Scrub on signs and symptoms of chronic blepharitis. DICP. 1991;25(4):359-360.

63. Guillon M, Maissa C, Wong S. Management of lid margin diseases with Blephaclean. Abstract 29 presented at 6th International Conference on the Tear Film and Ocular Surface: Basic Science and Clinical Relevance. September 22-25, 2010, Florence, Italy.

64. Doan S. Tolerability and acceptability of Blephagel: a novel eyelid hygiene aqueous gel. Clin Ophthalmol. 2012;6:71-77.

65. Gilbard JP, Douyon Y, Huson RB. Time-kill assay results for a linalool-hinokitiol-based eyelid cleanser for lid hygiene. Cornea. 2010;29(5):559-563.
Clinical Ophthalmology

\section{Publish your work in this journal}

Clinical Ophthalmology is an international, peer-reviewed journal covering all subspecialties within ophthalmology. Key topics include: Optometry; Visual science; Pharmacology and drug therapy in eye diseases; Basic Sciences; Primary and Secondary eye care; Patient Safety and Quality of Care Improvements. This journal is indexed on

\section{Dovepress}

PubMed Central and CAS, and is the official journal of The Society of Clinical Ophthalmology (SCO). The manuscript management system is completely online and includes a very quick and fair peer-review system, which is all easy to use. Visit http://www.dovepress.com/ testimonials.php to read real quotes from published authors. 